\title{
Atividade antimicrobiana de óleos essenciais produzidos por Baccharis dracunculifolia D.C. e Baccharis uncinella D.C. (Asteraceae)
}

\author{
Regina Ferronatto', Eli Danieli Marchesan', Emanueli Pezenti ${ }^{1}$, Franciela Bednarski', \\ Sideney Becker Onofre ${ }^{1,2 *}$
}

\author{
${ }^{1}$ Departamento de Biologia, Universidade Paranaense, UNIPAR, Caixa Postal 255, Bairro Industrial, 85601-000, \\ Francisco Beltrão, PR, Brasil, \\ ${ }^{2}$ União de Ensino do Sudoeste do Paraná, UNISEP, Avenida Presidente Kennedy, 2601, Bairro Nossa Senhora \\ Aparecida, 85660-000, Dois Vizinhos, PR, Brasil
}

\begin{abstract}
RESUMO: Os óleos essenciais obtidos de plantas nativas de Baccharis dracunculifolia e Baccharis uncinella, também conhecido como óleo-de-vassoura, é utilizado na indústria de perfumaria, proporcionando um aroma exótico a diversos perfumes, além de muitos estudos sobre atividades biológicas dessas espécies destacam os efeitos alelopáticos, antioxidante, antimicrobianos, citotóxicos e antiinflamatórios. A vassoura é uma planta arbustiva de ocorrência espontânea no Brasil, assim como nos demais países do Mercosul. A composição do óleo depende da região geográfica e do processo de extração utilizado e a importância comercial está diretamente relacionada com a concentração de compostos oxigenados, destacando o nerolidol e o espatulenol. Óleos essenciais obtidos dessas duas espécies pelo processo de hidrodestilação, foram avaliados pelo método de difusão em disco de papel, em placas de Petri contendo meio de Müeller-Hinton, semeadas com suspensões bacterianas previamente ajustadas ao tubo 0,5 da escala de Mac Farland de quatro cepas provenientes da American Type Culture Colection: Escherichia coli ATCC 25922, Staphylococcus aureus ATCC 25923 e Pseudomonas aeruginosa ATCC 27853. Discos contendo diferentes volumes dos óleos essenciais sem qualquer diluição (1, 3, 5 e $10 \mu \mathrm{L} /$ disco) foram adicionadas às placas e incubadas a $36^{\circ} \mathrm{C}$ por 24 a 48 horas. Discos de cloranfenicol (CLO) e amoxicilina (AMO) com volumes de 50 e $10 \mu \mathrm{g} /$ disco respectivamente, foram utilizados como controle. Os resultados apresentados revelam que ambos os óleos avaliados apresentam atividade antimicrobiana sobre S. aureus, E. coli e P. aeruginosa.
\end{abstract}

Unitermos: Baccharis, Asteraceae, atividade antimicrobiana, óleo essencial.

\begin{abstract}
Antimicrobial activity of essential oils produced by Baccharis dracunculifolia D.C. and Baccharis uncinella D.C. (Asteraceae)". Essential oils obtained from the native plants Baccharis dracunculifolia and Baccharis uncinella, known also as "óleo de vassoura”, are used in the perfumery industry, providing diverse perfumes with exotic aromas. Besides several studies about the biological activities of these species highlight the allelopathic, antioxidant, antimicrobial, cytotoxic and antiinflammatory effects. The broom is a bushy plant which occurs spontaneously in Brazil as well as in the other countries from Mercosul. The oil composition depends on the geographical region and on the concentration of oxygenated blends, highlighting the nerolidol and the sphatulenol. Essential oils obtained from these two species by the hydrodistillation process were assessed by the diffusion method in paper disc, in Petri plaques containing Müeller-Hinton agar, with bacteria suspensions, previously adjusted to the tube 0,5 of Mac Farland scale of four strains which came from the American Type Culture Collection: Escherichia coli ATCC 25922, Staphylococcus aureus ATCC 25923 and Pseudomonas aeruginosa ATCC 27853. Discs containing different volumes of the essential oils $(1,3,5$ and 10 ( $\mu \mathrm{L} /$ disc $)$ were added to plaques and incubated at $36{ }^{\circ} \mathrm{C}$ for 24 - 48 hours. Chloranfenicol (CLO) and Amoxicillin (AMO) with concentrations of 30 and $10 \mu \mathrm{g} /$ disc were respectively used as control. The presented results reveal that both assessed oils present antimicrobial activity against $S$. aureus, E. coli and P. aeruginosa.
\end{abstract}

Keywords: Baccharis, Asteraceae, antimicrobial activity, essential oil.

\section{INTRODUÇÃO}

Um dos maiores problemas de Saúde Pública, enfrentados nas últimas décadas foi o agravamento da resistência a antimicrobianos em populações bacterianas, principalmente de origem hospitalar (Fidler, 1998; Huovinen; Cars, 1998; Okeke et al., 1999; Oliveira et al., 2006a,b). Atualmente, registra-se um aumento 
significativo na freqüência do isolamento de bactérias que eram reconhecidamente sensíveis às drogas de rotina usadas na clínica, mas que se apresentam agora resistentes a todos ou quase todos os fármacos disponíveis no mercado, como ocorre com várias bactérias multirresistentes (Huycke et al., 1998; Rattan et al., 1998; Piddock et al., 1998; Pittet, 2002; Shiomori et al., 2002; Sakagami; Kajamura, 2006).

Muitas linhagens multirresistentes de Staphylococcus aureus - MRSA (Methicillin Resistant Staphylococcus aureus), por exemplo, reconhecido patógeno associado à infecção hospitalar ou adquirida na comunidade, já eram sensíveis apenas ao tratamento com vancomicina, mostrando-se resistentes aos aminoglicosídeos, $\quad \beta$-lactâmicos, macrolídeos, tetraciclinas, quinolonas e outros quimioterápicos (Salyers; Amábile-Cuevas, 1997, Chartone-Souza, 1998). Recentemente, entretanto, foi isolado tanto em laboratório (Di Sálvio, 1998) como em clínica (Domin, 1998) linhagens mutantes com sensibilidade reduzida à vancomicina. Obteve-se, inclusive, a transferência em laboratório de plasmídio $\mathrm{R}$, com determinante de resistência à vancomicina, para $S$. aureus, no qual felizmente o plasmídio não se estabilizou. Esses dados antecipam os gravíssimos problemas que poderão trazer essas linhagens multirresistentes, caso não surjam novos antimicrobianos ou terapias alternativas para combatê-los (Domin, 1998).

Atualmente, o problema da resistência tornou-se mais grave devido às dificuldades para a descoberta e o lançamento de novos antimicrobianos no mercado com o uso da metodologia tradicional de triagens, a partir de fungos e bactérias, o que vem tornando esses produtos cada vez mais escassos e mais caros (Phelps, 1989).

Estima-se que são necessários mais de 10 anos, a um custo superior a 200 milhões de dólares, para que um antimicrobiano esteja à disposição da medicina (Dickson; Redwood, 1998).

Uma das alternativas usadas pelas indústrias farmacêuticas tem sido a modificação química da estrutura dos antimicrobianos já existentes, na tentativa de torná-los mais eficientes ou de recuperar a atividade prejudicada pelos mecanismos bacterianos de resistência (Chartone-Souza, 1998). A mudança de paradigma pode ser uma das saídas futuras para o impasse, ao pesquisar em primeiro lugar o alvo e só depois preparar o princípio ativo contra ele, por técnicas de biologia molecular. Entretanto, as alternativas ecológicas, que enfatizam o respeito pelo meio ambiente, devem ser mais efetivas e de menor custo, uma vez que se baseiam no uso mais cuidadoso, e apenas quando necessário, levando à queda de pressão dos antimicrobianos de alguns ambientes, com a diminuição nos níveis atuais do seu uso. Por outro lado, torna-se relevante a pesquisa da ação inibitória do crescimento bacteriano ou do fluxo gênico de genes de resistência ou mesmo de reversão dessa resistência com o uso de produtos de origem natural (Linden; Paladino,

\section{8; Brown, 1999).}

$\mathrm{Na}$ busca de novos antimicrobianos devemos enfatizar aqueles de origem vegetal, uma vez que o Brasil apresenta a maior biodiversidade do planeta e que muitas plantas já vêm sendo vastamente usadas e testadas há centenas de anos com as mais diversas finalidades por populações do mundo inteiro. A maior parte da população brasileira (80\%) consome apenas 37\% dos medicamentos disponíveis, dependendo quase que exclusivamente de medicamentos de origem natural (Stangarlin et al., 1999; Funari; Ferro 2005). Para isso, antes da determinação das frações ativas por métodos bioquímicos sofisticados, agora disponíveis, torna-se necessário e útil o uso preliminar de métodos de triagem de domínio popular ou das farmácias homeopáticas como macerados, chás, extratos aquosos, alcoólicos e outros. Deve-se lembrar que os princípios ativos podem estar presentes em diferentes frações de um composto ou mesmo que um princípio ativo seja liberado só após a ação metabólica do animal (Carvalho, 2003).

Com o objetivo de conhecer as características químicas do gênero Baccharis, é que pesquisadores realizaram estudos com o gênero Baccharis para conhecê-las quimicamente e entre as estudadas, cerca de 30 apresentam estudos de atividade biológica.

A vassoura, Baccharis dracunculifolia (Asteraceae) é uma planta arbustiva que ocorre no Brasil, de Minas Gerais ao Rio Grande do Sul, nos países do Mercosul (Barroso, 1976) e nos vales elevados da Bolívia onde a planta é utilizada na medicina popular (Loayza et al., 1995). A composição do óleo tem sido estudada por vários autores Queiroga et al., 1990; (Loayza et al., 1993; 1995; Ferracini et al., 1995; Weyersthal et al., 1996).

Entre as espécies mais pesquisadas quanto à composição química e/ou atividade biológica, encontramse $B$. megapotamica, B. incarum, B. trimera, B. trinervis, B. salicifolia, B. uncinella B. crispa, B. coridifolia, $B$. dracunculifolia, B. grisebachii, B. genistelloides e B. tricuneata (Macari et al., 2002; Budel et al., 2004; Verdi et al., 2005; Budel et al., 2005; Sousa et al., 2007).

De um modo geral, os compostos que mais se destacaram nesses estudos, foram os flavonóides, clerodanos e labdanos, embora também se tenha observado com certa freqüência a presença de sesquiterpenos, diterpenos, triterpenos, saponinas, glicolipídios, flavonóides, ácidos cumáricos e fenilpropanóides (Queiroga et al., 1990; Borella et al., 2002; Zaroni et al., 2004; Borella et al., 2006; Mendes et al., 2006). Nos estudos de atividades biológicas evidenciam-se os efeitos alelopáticos, antimicrobianos, citotóxicos, hipoglicemiantes e antiinflamatórios (Loayza, et al., 1993, 1995; Duarte et al., 2004; Barbosa-Filho et al., 2005). Nesse sentido é que este trabalho buscou avaliar a atividade antimicrobiana dos óleos essenciais produzidos por B. uncinella e B. dracunculifolia coletados na região Sudoeste do Paraná. 


\section{MATERIAL E MÉTODOS}

Os óleos utilizados neste trabalho foram obtidos pelo processo de hidrodestilação utilizando para isso um extrator do tipo Clevenger. As espécies de Baccharis utilizadas para a obtenção dos óleos essenciais foram a B. dracunculifolia D.C. e B. uncinella D.C. (Asteraceae), coletadas na região Sudoeste do Estado do Paraná no período de janeiro a junho de 2005.

Os óleos obtidos foram avaliados utilizando o método de Kirby e Bauer (Vandepitte et. al., 1994) e o meio de cultivo foi o Müller e Hinton suplementado com 2\% de glicose. Uma suspensão bacteriana foi preparada em solução fisiológica estéril e ajustada ao tubo 0,5 da escala de McFarland, o que corresponde aproximadamente a $1,5 \times 10^{6} \mathrm{UFC} / \mathrm{mL}$ das cepas de Escherichia coli ATCC 25922 (beta-lactamase negativa) e Staphylococcus aureus ATCC 25923 (suscetível à oxacilina e penicilina), e Pseudomonas aeruginosa ATCC 27853, de propriedade do Laboratório de Microbiologia da Universidade Paranaense, Campus de Francisco Beltrão.

Alíquotas de $100 \mu \mathrm{L}$ de cada suspensão bacteriana foram semeadas por superfície em placas de Petri contendo cerca de $15 \mathrm{~mL}$ do meio MuellerHinton, com uma espessura de aproximadamente $4 \mathrm{~mm}$ (Shadomy; Spnel-Ingrof, 1980).

Discos de papel-filtro de seis milímetros de diâmetro, esterilizados contendo volumes de 1, 3, 5 e $10 \mu \mathrm{L}$ de óleo puro, sem qualquer diluição, de cada espécie avaliada foram colocados sobre as suspensões bacterianas nas placas de Petri. O teste-controle foi realizado com discos dos antibióticos cloranfenicol (CLO) e amoxilina (AMO) contendo $50 \mu \mathrm{g}$ e $10 \mu \mathrm{g}$ por disco, respectivamente, disponíveis comercialmente ${ }^{\circledast}$.

A incubação foi feita em estufa a $36{ }^{\circ} \mathrm{C}$ durante 24 a 48 horas. Os testes foram realizados em triplicata e os resultados expressos em mm pela média aritmética do diâmetro dos halos de inibição formado ao redor dos discos nas 3 repetições.

Os diâmetros dos halos de inibição foram interpretados de acordo com os critérios de interpretação preconizados pelo NCCLS (National Committee for Clinical Laboratory Standards, 1997, 2000a e 2000b).

As análises de variância foram realizadas segundo normas da ANOVA. As diferenças significativas entre as médias foram determinadas pelo teste de Tukey.

\section{RESULTADOS E DISCUSSÃO}

Estudos indicam que óleos essenciais têm efeito bactericida contra muitas bactérias (Nenof et al., 1996; Kalpoutzakis et al., 2001). Segundo Ohno et al., 2003, há diferenças na atividade bactericida entre os óleos essenciais, embora a razão exata ainda não tenha sido esclarecida. Os resultados encontrados no presente estudo estão em concordância com os desses pesquisadores. Conforme relata Koyama et al. 1997, muitos componentes dos óleos essenciais, os quais são diferentes em cada óleo, possuem uma habilidade específica para romper ou penetrar na estrutura bacteriana. Para se conhecer o modo de ação dos óleos seria necessário examinar separadamente cada componente do óleo essencial, e a combinação para averiguar se eles têm ação bactericida sozinhos ou sincronizados.

Os resultados obtidos neste trabalho estão sumarizados nas Tabelas 1 e 2 e na Figura 1. Em análise dos resultados obtidos verificamos que ambos os óleos mostraram alguma atividade inibitória sobre o crescimento microbiano das cepas testadas.

Quando foi analisado os resultados obtidos com o óleo de $B$. uncinella (Tabela 1), foi verificado que este óleo inibiu o crescimento de $E$. coli nos volumes de 1 , 3,5 e $10 \mu \mathrm{L}$ do óleo puro por disco, induzindo a formação de halos inibição, respectivamente, de 8,22 mm, 8,31 mm, $9,12 \mathrm{~mm}$, e 13,05 mm. Para S. aureus, os halos formados, para os mesmos volumes do óleo por disco, foram de $9,46 \mathrm{~mm}, 10,83 \mathrm{~mm}, 12,40 \mathrm{~mm}$ e $14,68 \mathrm{~mm}$; para $P$. aeruginosa, este óleo mostrou atividade apenas com 10 $\mu \mathrm{L}$ do óleo puro de $B$. uncinella, apresentando um halo de inibição de 7,32 mm, mostrando com isso uma alta resistência desse microrganismo a ação dos componentes presentes no óleo.

Comparando os resultados obtidos pelo óleo de B. uncinella em relação aos discos de antibióticos controle, AMO e CLO, foi possível verificar resultados significativos tendo em vista que com o volume de $10 \mu \mathrm{L}$ do óleo puro, foram obtidos 68,25 \%, 79,73 \% e 76,97 \% de inibição, em relação aos discos de CLO $30 \mu \mathrm{g} /$ disco e de $80,40 \%, 51,83 \%$ e $77,75 \%$ quando comparados com discos de AMO $10 \mu \mathrm{g} /$ disco respectivamente, sobre E. coli, S. aureus e P. aeruginosa.

O óleo obtido de B. dracunculifolia apresentou resultados semelhantes (Tabela 2) aqueles obtidos com o óleo de B. uncinella. Esses dados analisados mostram halos de inibição do crescimento de E. coli mostrou variações entre 9,25 mm para $1 \mu \mathrm{L}$ a 13,62 mm para 10 $\mu \mathrm{L}$ do óleo puro adicionado aos discos. Para $S$. aureus essa variação foi de $8,35 \mathrm{~mm}$ para $1 \mu \mathrm{L}$ a $14,63 \mathrm{~mm}$ para $10 \mu \mathrm{L}$. Este óleo apresentou uma baixa atividade inibitória sobre $P$. aeruginosa, verificando que para os volumes de 1,3 e $5 \mu \mathrm{L}$ a mesma se mostrou resistente. Apenas para $10 \mu \mathrm{L}$ foi verificado uma pequena sensibilidade com formação de um halo de inibição de 8,32 mm.

A comparação dos resultados obtidos pelo óleo de B. dracunculifolia em relação aos discos de antibióticos controle, AMO e CLO, mostrou resultados significativos onde com o volume de $10 \mu \mathrm{L}$ do óleo puro, obteve-se $93,61 \%, 84,03 \%$, e $81,48 \%$ de inibição, em relação aos discos de CLO $30 \mu \mathrm{g} /$ disco e de $24,22 \%$, $53,39 \%$ e $58,46 \%$ quando comparados com discos de AMO $10 \mu \mathrm{g} / \mathrm{disco}$, respectivamente, sobre E. coli, S. aureus e $P$. aeruginosa. Nesta comparação foi possível verificar que neste trabalho, nas condições utilizadas e por métodos aqui descritos, seguindo a metodologia oficial 
Tabela 1. Diâmetros dos halos de inibição em mm do óleo essencial obtido de B. uncinella, coletados na região Sudoeste do Paraná.

\begin{tabular}{l|c|c|c|c|c|c}
\hline Microrganismo & $1 \mu \mathrm{L}$ & $3 \mu \mathrm{L}$ & $5 \mu \mathrm{L}$ & $10 \mu \mathrm{L}$ & CLO & AMO \\
\hline E. coli & $8,22 \mathrm{a}^{*}$ & $8,31 \mathrm{a}$ & $9,12 \mathrm{a}$ & $13,05 \mathrm{~b}$ & $19,12 \mathrm{c}$ & $16,23 \mathrm{c}$ \\
S. aureus & $9,46 \mathrm{a}$ & $10,83 \mathrm{a}$ & $12,41 \mathrm{ab}$ & $14,68 \mathrm{~b}$ & $18,41 \mathrm{c}$ & $28,32 \mathrm{~d}$ \\
P. aeruginosa & 0,00 & 0,00 & 0,00 & $7,32 \mathrm{a}$ & $9,51 \mathrm{a}$ & $12,23 \mathrm{a}$ \\
\hline
\end{tabular}

*Médias seguidas pela mesma letra, não diferem entre si, pelo teste de Tukey, a 5\% de probabilidade.

Tabela 2. Diâmetros dos halos de inibição em mm do óleo essencial obtido de B. dracunculifolia, coletados na região Sudoeste do Paraná.

\begin{tabular}{l|c|c|c|c|c|c}
\hline Microrganismo & $1 \mu \mathrm{L}$ & $3 \mu \mathrm{L}$ & $5 \mu \mathrm{L}$ & $10 \mu \mathrm{L}$ & CLO & AMO \\
\hline E. coli & $9,25 \mathrm{a}^{*}$ & $13,62 \mathrm{~b}$ & $15,05 \mathrm{~b}$ & $18,92 \mathrm{c}$ & $20,21 \mathrm{c}$ & $15,23 \mathrm{c}$ \\
S. aureus & $8,35 \mathrm{a}$ & $8,76 \mathrm{a}$ & $13,31 \mathrm{~b}$ & $14,63 \mathrm{~b}$ & $17,41 \mathrm{c}$ & $27,40 \mathrm{~d}$ \\
P. aeruginosa & 0,00 & 0,00 & 0,00 & $8,32 \mathrm{a}$ & $10,21 \mathrm{ab}$ & $14,23 \mathrm{~b}$ \\
\hline
\end{tabular}

*Médias seguidas pela mesma letra, não diferem entre si, pelo teste de Tukey, a 5\% de probabilidade.

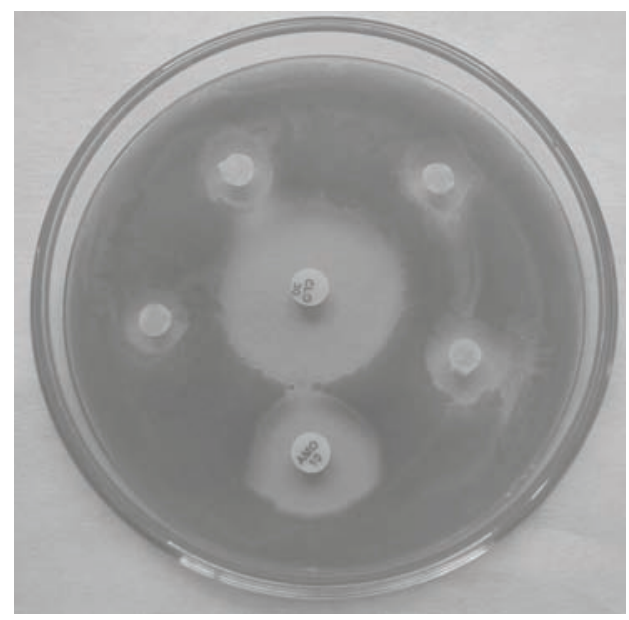

Figura 1. Atividade antimicrobiana dos óleo essencial de B. uncinella, a $1 \mu \mathrm{L}$ (1), $3 \mu \mathrm{L}$ (2), $5 \mu \mathrm{L}$ (3), $10 \mu \mathrm{L}$ (4) e controles com amoxilina (AMO) e cloranfenicol (CLO) sobre E. coli ATCC 25922 (beta-lactamase negativa).

(Vandepitte et. al., 1994), com o volume de $10 \mu \mathrm{L} /$ disco do óleo puro de B. dracunculifolia, um comportamento 24\% superior ao disco de AMO $10 \mu \mathrm{g} /$ disco.

A partir dos resultados obtidos, verificou-se que o comportamento desses dois óleos quando avaliados pela metodologia adotada, se mostraram semelhantes, o que sugere que o modo de ação, os componentes químicos e as respectivas concentrações, também são semelhantes.

Os resultados obtidos neste trabalho são inéditos para B. dracunculifolia e complementam os de B. uncinella descritos na literatura especializada referente a essas duas espécies (Agostini et al., 2005). Outros trabalhos com outras espécies de Baccharis mostram atividade antimicrobiana. Em Baccharis trimera, Avancini et al. (2000), confirmaram a atividade antimicrobiana sobre $S$. aureus e E. coli. Em estudos realizados por Rangel et al. (2001), avaliando a atividade antimicrobiana de Baccharis nitida, os autores observaram atividade antimicrobiana sobre as bactérias $S$. aureus e $P$. aeruginosa, sendo bactérias Gram-positiva e Gramnegativa respectivamente, coincidindo com os resultados obtidos nesse trabalho.

Segundo Verdi et al., 2005, o uso de plantas para curar doenças, incluindo as infecciosas, tem sido extensivamente aplicada pelas pessoas, informações da literatura e os seus resultados revelam um grande potencial das plantas para tratamentos terapêuticos, apesar do fato que elas não tenham sido completamente estudadas, mais estudos precisam ser conduzidos para pesquisar sobre novos componentes. Segundo Verdi et al. 2005, a atividade antimicrobiana tem sido encontrada com alguns terpenos originados de plantas. Uma vez extraídos, e antes de serem usados como tratamento terapêutico, eles devem ter a toxicidade testada in vivo, 
testes têm demonstrado a toxicidade de extratos de diferentes plantas.

O estudo das plantas medicinais, como ficaram conhecidas no mundo todo pelos pesquisadores, está sendo desenvolvido, comprovando as enormes qualidades medicinais das mesmas, sejam de outros países, ou das milhares de espécies espalhadas pelos quatro cantos do país e em outras partes do mundo que, ao mesmo tempo em que vêm ganhando respeito e maior espaço nas prateleiras de farmácias e casas especializadas, também vêm apresentando propriedades nunca antes exploradas (Amoroso; Gely, 1988; Brandão et al., 2001; Funari; Ferro 2005; Ribeiro et al., 2005; Silva et al., 2006). Além disso, a grande incidência de infecções, principalmente em indivíduos imunocomprometidos, aumenta a importância da procura e descoberta de compostos terapêuticos alternativos (Carriconde et al., 1996).

\section{CONCLUSÃO}

De acordo com os resultados obtidos, utilizando a metodologia oficial, podemos concluir que os óleos essenciais obtidos de B. uncinella e B. dracunculifolia, quando avaliados sobre as bactérias patogênicas Escherichia coli (ATCC 25922-beta-lactamase negativa), Pseudomonas aeruginosa (ATCC 27853) e Staphylococcus aureus (ATCC 25923-suscetível a oxacilina e penicilina), mostraram-se eficientes em inibir o crescimento dos microrganismos testados. Com os resultados obtidos, podemos sugerir que estudos posteriores sejam realizados buscando caracterizar quimicamente os óleos estudados e posteriormente identificar os componentes desses óleos e quais grupamentos químicos apresentam essa atividade sobre as bactérias testadas.

\section{REFERÊNCIAS}

Agostini F, Santos ACA, Rossato M, Pansera MR, Zattera F, Wasum R, Serafini LA 2005. Estudo do óleo essencial de algumas espécies do gênero Baccharis (Asteraceae) do sul do Brasil. Rev Bras Farmacogn 15: 215-220.

Amorozo MCM, Gely A 1988. Uso de plantas medicinais por caboclos do Baixo Amazonas; Barcarena: PA. Museu Paraense Emílio Goeldi 4: 47.

Avancini CAM, Wiest JM, Mundstock E 2000. Bacteriostatic and bactericidal activity of the Baccharis trimera (Less.) D.C. - Compositae decocto, as disinfectant or antisseptic. Arq Bras Med Vet Zootec 52: 230-234.

Barbosa-Filho JM, Vasconcelos THC, Alencar AA, Batista LM, Oliveira RAG, Guedes DN, Falcão HS, Moura MD, Diniz MFFM, Modesto-Filho J 2005. Plants and their active constituents from South, Central, and North America with hypoglycemic activity. Rev Bras Farmacogn 15: 392-413.

Barroso GM 1976. Compositae-Subtribo Baccharidinae Hoffmann. Estudo das espécies ocorrentes no Brasil.
Rodriguésia 35: 26-32.

Borella JC, Fontoura A 2002. Avaliação do perfil cromatográfico e do teor de flavonóides em amostras de Baccharis trimera (Less) DC., Asteraceae (carqueja) comercializadas em Ribeirão Preto, SP, Brasil. Rev Bras Farmacogn 12: 63-67.

Borella JC, Duarte DP, Novaretti AAG, Menezes Jr A, França SC, Rufato CB, Santos PAS, Veneziani RCS, Lopes NP 2006. Variabilidade sazonal do teor de saponinas de Baccharis trimera (Less.) DC (Carqueja) e isolamento de flavona. Rev Bras Farmacogn 16: 557-561.

Brandão MGL, Moreira RA, Acúrcio FA 2001. Interesse dos estudantes de Farmácia e Biologia por plantas medicinais e fitoterapia. Rev Bras Farmacogn 11: 7176.

Brown C 1999. Resistance mechanisms to drugs. In: Brown R, Boger-Brown U, editors. Cytotoxic drug resistance mechanisms. Totowa: Humana Press p. 30-59.

Budel JM, Duarte MR, Santos CAM 2004. Parâmetros para análise de carqueja: comparação entre quatro espécies de Baccharis spp. (Asteraceae). Rev Bras Farmacogn 14: 41-48.

Budel JM, Duarte MR, Santos CAM, Farago PV, Matzenbacher NI 2005. O progresso da pesquisa sobre o gênero Baccharis, Asteraceae: I - Estudos botânicos. Rev Bras Farmacogn 15: 268-271.

Carriconde C, Mores D, Von Fritschen M, Cardozo-Junior EL 1996. Plantas medicinais e alimentícias. Olinda: Centro Nordestino de Medicina Popular; Universidade Federal Rural de Pernambuco. v.1, p.45-47.

Carvalho I 2003. Introduction to molecular modeling of drugs in the medicinal chemistry experimental course. Quim Nova 26: 428-438.

Chartone-Souza E 1998. Bactérias ultra-resistentes: uma guerra quase perdida. Ciência Hoje 23: 26-35.

Di Sálvio VMP 1998. Estudo da resistência a drogas em Staphylococcus aureus de origem hospitalar $e$ obtenção de mutantes resistentes à vancomicina. Belo Horizonte: UFMG, 1998. Dissertação de Mestrado em Microbiologia, Universidade Federal de Minas Gerais.

Dickson M Redwood H 1998. Pharmaceutical reference prices. How do they work in practice? Pharmacoeconomics 14: 471-479.

Domin MA 1998. Highly virulent pathogens-a post antibiotic era. Br J Theatre Nurs 8: 14-18.

Duarte MCT, Figueira GM, Pereira B, Magalhães PM, Delarmelina C 2004. Atividade antimicrobina de extratos hidroalcoólicos de espécies da coleção de plantas medicinais CPQBA/UNICAMP. Rev Bras Farmacogn 14(Supl. 1): 6-8.

Ferracini VL, Paraiba LC, Leitao Filho HF, Silva AG, Nascimento LR, Marsaioli A 1995. Essential oil of seven Brazilian Baccharis sp. .J Essent Oil Res 7: 355-367.

Fidler DP 1998. Legal issues associated with antimicrobial drug resistance. Emerg Infect Dis 4: 169-177.

Funari CS, Ferro VO 2005. Uso ético da biodiversidade 
brasileira: necessidade e oportunidade. Rev Bras Farmacogn 15: 178-182.

Huovinen P, Cars O 1998. Control of antimicrobial resistance: time for action. Braz Med J 317: 613-615.

Huycke MM, Sahm DF, Gilmore MS 1998. Multiple-drug resistant Enterococci: The nature of the problem and the agenda for the future. Emerg Infect Dis 4: 239249.

Kalpoutzakis E, Aligiannis N, Mentis A, Mitaku S, Charvala C 2001. Composition of the essential oil of two nepeta species and in vitro evaluation of their activity against Helicobacter pylori. Planta Med 67: 880-883.

Koyama S, Yamaguchi Y, Tanaka S, Motoyashima J 1997. A new substance (yoshixol) with na interesting antibiotic mechanism from wood oil of Japanase traditional tree (kisohinoki), Chamaecyparis obtusa. Gen Pharmacol 28: 797-804.

Linden P, Paladino JA 1998. The economic impact of bacteremia due to vancomycin-resistant Enterococcus faecium: a case-control study. 38th Interscience Conference on Antimicrobial Agents and Chemotherapy. San Diego, CA. Sep 24-27.

Loayza I, Abujder D, Aranda R, Jakupovic J, Collin G, Deslauriers HE, Jean FI 1995. Essential oils of Baccharis salicifolia, Baccharis latifolia and Baccharis dracunculifolia. Phytochemistry 38: 381389.

Loayza I, Collin G, Gagnon M, Deslauriers H, Dellacassa E 1993. Huiles essentielles de Baccharis latifolia, $B$. salicifolia de Bolivia de $B$. dracunculifolia en provenance d'Uruguay”. Rivista Ital EPPOS Ed. Especial: 728-735.

Macari PAT, Sousa RR, Crespo MLL, Martins PA, Vieira JDD, Velosa AC, Fávero O, Gomes EPC 2002. Comparação entre os metais presentes em Croton floribundus Spreng. e Baccharis dracunculifolia DC. Rev Bras Farmacogn 12(Supl. 1): 76-77.

Mendes BG, Machado MJ, Falkenberg M 2006. Triagem de glicolipídios em plantas medicinais. Rev Bras Farmacogn 16: 568-575.

NCCLS 1997. National Committee for Clinical Laboratory Standards. Approved standard M2-A7: Performance standards for antimicrobial disk susceptibility tests. Seventh edition. Wayne, PA.

NCCLS 2000a. National Committee for Clinical Laboratory Standards. Approved standard M7-A5: Methods for dilution antimicrobial susceptibility test for bacteria that grow aerobicaly. Fifth edition. Wayne, PA.

NCCLS 2000b. National Committee for Clinical Laboratory Standards. Performance standards for antimicrobial suceptibility testing; tenth informational supplement M100-S10. Wayne, PA.

Nenoff P, Haustein UF, Brandt W 1996. Antifungal activity of the essential oil Melaleuca alternifolia (tea tree oil) against pathogenic fungi in vitro. Skin Pharmacol 9: 388-394.

Ohno T, Kita M, Yamaoka Y, Imamura S, Yamamoto SM,
Kodama T, Kashima K, Imanishi J 2003. Antimicrobial activity of essential oils against Helicobacter pylori. Helicobacter 8: 207-215.

Okeke IN, Lamikanra A, Edelman R 1999. Socioeconomic and behavioral factors leading to acquired bacterial resistance to antibiotics in developing countries. Emerg Infect Dis 5: 18-27.

Oliveira RAG, Lima EO, Vieira WL, Freire KRL, Trajano VN, Lima, IO, Souza EL, Toledo MS, Silva-Filho RN 2006. Estudo da interferência de óleos essenciais sobre a atividade de alguns antibióticos usados na clínica. Rev Bras Farmacogn 16: 77-82.

Oliveira FP, Lima EO, Siqueira Júnior JP, Souza EL, Santos BHC, Barreto HM 2006. Effectiveness of Lippia sidoides Cham. (Verbenaceae) essential oil in inhibiting the growth of Staphylococcus aureus strains isolated from clinical material. Rev Bras Farmacogn 16: $510-516$.

Phelps CE 1989. Bug/drug resistance: sometimes less is more. Med Care 27: 194-203.

Piddock LJV, Ricci V, Mclaren I, Griggs DJ 1998. Role of mutation in the gyrA and parC genes of nalidixic-acidresistant Salmonella serotypes isolated from animals in United Kingdom. J Antimicrobial Chemother 41: 635-641.

Pittet D 2002. Promotion of hand hygiene: magic, hype, or scientific challenge? Infect Control Hosp Epidemiol 23: 118-119.

Queiroga CL, Fukai AE, Marsaioli A 1990. Composition of the essential oil of vassoura. J Braz Chem Soc 1: 105109.

Rangel D, García I, Velasco J, Buitragro D, Velazco E 2001. Actividad antimicrobiana de los extractos etanólico, acetónico y acuoso de Baccharis nitida. Revista de la Faculdad de Farmacia 42: 35-46.

Rattan A, Kalia A, Ahmad N 1998. Multidrug-resistant Mycobacterium tuberculosis: Molecular perspectives. Emerg Infec Dis 4: 195-209.

Ribeiro AQ, Leite JPV, Dantas-Barros AM 2005. Perfil de utilização de fitoterápicos em farmácias comunitárias de Belo Horizonte sob a influência da legislação nacional. Rev Bras Farmacogn 15: 65-70.

Sakagami Y, Kajamura K 2006. Bactericidal activities of desinfectants against vancomycin-resistant Enterococci. J Hosp Infect 56: 140-144.

Salyers AA, Amábile-Cuevas CF 1997. Why are antibiotic genes so resistant to elimination? Antimicrobial Agents Chemother 41: 2321-2325.

Shadomy S, Spinel-Ingrof A 1980. Susceptibility testing: with antifungal drugs. In: Lennette, E. Manual of clinical microbiology. 3.ed. Washington: Americam Society for Microbiology p. 647-653.

Shiomori T, Miyamoto H, Makashima K, Yoshida M, Fujiyoshi T, Udaka T, Inaba T, Hiraki N 2002. Evaluation of bedmaking-related airborne and surface methicillinresistant Staphylococcus aureus contamination. J Hosp Infect 50: 30-35. 
Silva MIG, Gondim APS, Nunes IFS, Sousa FCF 2006. Utilização de fitoterápicos nas unidades básicas de atenção à saúde da família no município de Maracanaú (CE). Rev Bras Farmacogn 16: 455-462.

Sousa JPB, Furtado NAJC, Jorge R, Soares AEE, Bastos JK 2007. Perfis físico-químico e cromatográfico de amostras de própolis produzidas nas microrregiões de Franca (SP) e Passos (MG), Brasil. Rev Bras Farmacogn 17: 85-93.

Stangarlin RJ, Schwan-Estrada KRF, Nozaki MH 1999. Plantas medicinais e controles alternativos de fitopatógenos. Biotecnol Cienc Des 11: 16-21.

Vandepitte J, Engbaek K, Piot PE, Heuck CC 1994. Procedimentos laboratoriais em bacteriologia clínica. OMS. Editora Santos, São Paulo. p.87.

Verdi LG, Brighente IMC, Pizzolatti MG 2005. O gênero Baccharis (Asteraceae): Aspectos químicos, econômicos e biológicos. Quim Nova 28: 85-94.

Weyerstahl P, Christiansen C, Marschall H 1996. Constituents of Brazilian vassoura oil. Flavour Frag J 11: 15-23.

Zaroni M, Pontarolo R, Abrahão WSM, Fávero MLD, CorreaJúnior C, Stremel DP 2004. Qualidade microbiológica das plantas medicinais produzidas no Estado do Paraná. Rev Bras Farmacogn 14: 29-39. 